\title{
Opérateur de Hardy et espaces Gaussiens
}

\author{
Youssef Ouknine, Youssef Elhssaini \\ Faculté des Sciences Semlalia \\ Département de Mathématiques \\ Université Cadi Ayyad \\ BP 2390, Marrakech, Maroc
}

Mai 2004

\section{Résumé}

Ce travail est une extention du résultat du deuxième paragraphe dans [2] (et par suite celui de [4]), où on a démontré une représentation Chaotique pour le pont Brownien généralisé; En fait nous allons montrer ici un résultat simailaire pour le pont généralisé du drap Brownien à $n$ paramétres .

Mots clés : Opérateur de Hardy, Pont généralisé, Drap Brownien, Formule de Stroock.

AMS Subject classification : 60G99,60H05, 60J65

\section{Introduction}

Soit $X(t)$ un drap brownien indexé par l'ensemble $C_{n}:=[0,1]^{n}$ et défini sur un espace probabilisé $(\Omega, \mathcal{F}, \mathcal{P}) ;$ Autrement dit, $X(\mathbf{t}), \mathbf{t} \in C_{n}$ est une famille de gaussiennes centrées telles que, pour tout $\mathbf{t}=\left(t_{1}, \cdots, t_{n}\right)$ et $\mathbf{s}=\left(s_{1}, \cdots, s_{n}\right) \in C_{n}$ on a :

$$
\mathbb{E}(X(\mathbf{t}) X(\mathbf{s}))=\prod_{i=1}^{n} t_{i} \wedge s_{i}
$$

Pour $\mathbf{u}=\left(u_{1}, \cdots, u_{n}\right) \in C_{n}$ on définit :

$$
R_{u}:=\left[0, u_{1}\right] \times\left[0, u_{2}\right] \cdots \times\left[0, u_{n}\right] \text { et } \partial R_{u}:=R_{u}-\left[0, u_{1}\right) \times\left[0, u_{2}\right) \cdots \times\left[0, u_{n}\right)
$$

On désigne par $\mathcal{C}\left(R_{u}\right)$ et $\mathcal{C}\left(\partial R_{u}\right)$ la classe des fonctions continues qui s'annulent sur les axes et définies respectivement sur $R_{u}$ et $\partial R_{u}$. Le long de ce travail nous allons définir une classe de processus $\left\{\left(X_{n, u}^{g, y}\right), y \in \mathcal{C}\left(\partial R_{u}\right)\right\}$ indexé par $C_{n}$ telle que la loi de cette famille notée par $\left\{\mathbb{P}_{n, u}^{g, y}, \quad y \in \mathcal{C}\left(\partial R_{u}\right)\right\}$ est faiblement continue (en y).

Etant donné un processus $Y(\mathbf{t})$ indexé par $C_{n}$ et ayant la loi $\mathbb{P}_{n, u}^{g, y}$ pour un certain $y \in \mathcal{C}\left(\partial R_{u}\right)$ donnée, on dira que c'est un pont du drap Brownien sur $R_{u}$, et on montre que la classe des variables aléatoire

$$
\left\{\int f(\mathbf{t}) Y(d \mathbf{t}): f \in L^{2}\left(C_{n}, d \lambda_{n}\right)\right\}
$$

où, $\lambda_{n}$ est la mesure de Lebesgue, est bien définie.

Ce travail est constitué de deux paragraphes : le premier paragraphe contient quelques résultats sur les processus gaussienns à $\mathrm{n}$ paramètres introduisant l'opérateur de Hardy généralisé, quand au deuxième, c'est une extention du résultat de Gosselin et Wurzbacher [4] concernant la représentation Chaotique du pont Brownien; Plus précisement, on montre un résultat similaire pour le pont généralisé du drap Brownien à n paramètres indexé par $C_{n} n>1$. 


\section{Pont Brownien généralisé et Opérateur de Hardy généralisé.}

Dans cette section nous allons définir des opérateurs un peu plus généraux que ceux définis dans le premier paragraphe de [2]. Pour cela, on consédère l'ensemble $C_{n}=[0,1]^{n} n \geq 1$, et pour un certain $\mathbf{u}=\left(u_{1}, \cdots, u_{n}\right) \in C_{n}$, on prend $R_{u}$ et $\partial R_{u}$ comme dans (1) et $\lambda_{n}$ la mesure de Lebesgue sur $C_{n}$. Nous allons introduire les deux ensembles suivants :

$$
\begin{aligned}
L^{(n)} & =L^{2}\left(C_{n}, \lambda_{n}\right) \\
L_{g}^{(\mathbf{u}, n)} & =\left\{f \in L^{(n)} ; \int_{R_{t}} f g^{\otimes^{n}} d \lambda_{n}=0 ; \forall \mathbf{t} \in \partial R_{u}\right\}
\end{aligned}
$$

où $g \in L^{2}([0,1])$ et vérifie en plus la condition :

$$
\int_{0}^{u_{i}} \frac{|g(s)|}{\sqrt{\int_{s}^{u_{i}} g^{2}(r) d r}} d s<+\infty \quad \forall i=1, \cdots, n
$$

Le produit scalaire et la norme seront notés respectivement $(,)_{n}$ et $\|\cdot\|_{n}$.

Pour la suite on considère les deux familles de gaussiennes centrées suivantes :

$$
\begin{aligned}
X_{n} & :=\left\{X_{n}(f), f \in L^{(n)}\right\} \\
X_{n, u}^{g} & :=\left\{X_{n, u}^{g}(f), f \in L_{g}^{(u, n)}\right\} .
\end{aligned}
$$

telles que :

$$
\begin{aligned}
\mathbb{E}\left(X_{n}(f) X_{n}(h)\right) & =(f, h)_{n} \quad f, h \in L^{(n)} \\
\mathbb{E}\left(X_{n, u}^{g}(f) X_{n, u}^{g}(h)\right) & =(f, h)_{n} \quad f, h \in L_{g}^{(u, n)},
\end{aligned}
$$

Un processus $X_{n}$ de ces deux familles sera appelé respectivement un processus gaussien isonormal dans $L^{(n)}\left(\operatorname{resp} . L_{g}^{(u, n)}\right)$.

Nous allons maintenant introduire les deux opérateurs suivants, qui sont, à titre de rappel, les mêmes que nous avons défini dans [2]. Pour $f \in L^{2}([0,1]), g \in L^{2}([0,1])$ vérifiant $(\star)$ et $u \in[0,1]$ on définit :

$$
\begin{aligned}
T_{g}^{(u)} f(s) & =f(s)-g(s) \frac{\int_{s}^{u} g(r) f(r) d r}{\int_{s}^{u} g^{2}(r) d r} 1_{(s<u)} . \\
S_{g}^{(u)} f(s) & =f(s)-g(s) \int_{0}^{s} \frac{g(r) f(r)}{\int_{r}^{u} g^{2}(v) d v} d r 1_{(s<u)} . \\
R_{g}^{(u)} f(s) & =f(s)-\frac{\left(f 1_{(0, u)}, g\right)}{\left\|g 1_{(0, u)}\right\|_{2}^{2}} g(s) 1_{(s<u)} .
\end{aligned}
$$

Notons $C_{n}^{+}=\left\{\mathbf{u} \in C_{n} ; u_{i}>0 \forall i=1, \cdots, n\right\}$, et considèrons les opérateurs suivants : Pour tout $\mathbf{u} \in C_{n}^{+}$et $f \in L^{(n)}$

$$
\begin{aligned}
T_{g}^{(\mathbf{u}, n)} f & :=T_{(g, 1)}^{\left(u_{1}\right)}\left[T_{(g, 2)}^{\left(u_{2}\right)} \cdots\left(T_{(g, n)}^{\left(u_{n}\right)} f\right)\right] \\
S_{g}^{(\mathbf{u}, n)} f & :=S_{(g, 1)}^{\left(u_{1}\right)}\left[S_{(g, 2)}^{\left(u_{2}\right)} \cdots\left(S_{(g, n)}^{\left(u_{n}\right)} f\right)\right] \\
R_{g}^{(\mathbf{u}, n)} f & :=R_{(g, 1)}^{\left(u_{1}\right)}\left[R_{(g, 2)}^{\left(u_{2}\right)} \cdots\left(R_{(g, n)}^{\left(u_{n}\right)} f\right)\right]
\end{aligned}
$$

où $T_{(g, n)}^{\left(u_{n}\right)}$ est l'opérateur qui agit sur $f$ comme étant fonction uniquement de la nième variable en regardant tous les autres fixes. Un premier résultat qui explique la relation entre les trois objets dans (6) se résume dans la proposition suivante : 
Proposition 1 Pour $n \geq 1$ fixé, soit $\mathbf{u}=\left(u_{1}, \cdots, u_{n}\right) \in C_{n}^{+}$et soit $T_{g}^{(\mathbf{u}, n)}, S_{g}^{(\mathbf{u}, n)}$ et $R_{g}^{(\mathbf{u}, n)}$ comme dans (6). Alors on a:

i) L'opérateur $R_{g}^{(\mathbf{u}, n)}$ est une application de $L^{(n)}$ dans $L_{g}^{(\mathbf{u}, n)}$ et sa restriction à $L_{g}^{(\mathbf{u}, n)}$ coincide avec l'opérateur identité.

ii) L'opérateur $T_{g}^{(\mathbf{u}, n)}\left(\operatorname{resp} S_{g}^{(\mathbf{u}, n)}\right)$ est un isomorphisme de $L_{g}^{(\mathbf{u}, n)}\left(\operatorname{resp} L^{(n)}\right)$ dans $L^{(n)}\left(\operatorname{resp} L_{g}^{(\mathbf{u}, n)}\right)$ iii) Pour tout $f \in L^{(n)}$ on $a$ :

$$
T_{g}^{(\mathbf{u}, n)}\left(R_{g}^{(\mathbf{u}, n)} f\right)=T_{g}^{(\mathbf{u}, n)} f \quad \text { et } \quad S_{g}^{(\mathbf{u}, n)}\left(T_{g}^{(\mathbf{u}, n)} f\right)=R_{g}^{(\mathbf{u}, n)} f .
$$

Peuve :

pour montrer (i) il suffit de le faire pour $n=2$, sachant que pour $n=1$ ce n'est que le lemme I.2.1 dans Jeulin et Chaleyat Maurel [6].

En effet, soit $\mathbf{u}=\left(u_{1}, u_{2}\right) \in[0,1]^{2}$ et $f \in L^{(2)}$ alors on a :

$$
\begin{aligned}
R_{g}^{(\mathbf{u}, 2)} f(s, t)= & f(s, t)-\frac{\int_{0}^{u_{1}} f(r, t) g(r) d r}{\left\|g 1_{\left(0, u_{1}\right)}\right\|_{2}^{2}} g(s) 1_{\left(s<u_{1}\right)} \\
& -\frac{\int_{0}^{u_{2}} f(s, r) g(r) d r}{\left\|g 1_{\left(0, u_{2}\right)}\right\|_{2}^{2}} g(t) 1_{\left(t<u_{2}\right)}+\frac{\int_{0}^{u_{1}} \int_{0}^{u_{2}} f(r, v) g(r) g(v) d r d v}{\left\|g 1_{\left(0, u_{2}\right)}\right\|_{2}^{2}\left\|g 1_{\left(0, u_{1}\right)}\right\|_{2}^{2}} g(s) g(t) 1_{\left(s<u_{1}\right)} 1_{\left(t<u_{2}\right)}
\end{aligned}
$$

donc pour tout $a \in[0,1]$ on a :

$$
\begin{aligned}
\int_{0}^{a} \int_{0}^{u_{1}} R_{g}^{(\mathbf{u}, 2)} f(s, t) g(s) g(t) d s d t= & \int_{0}^{a} \int_{0}^{u_{1}} f(s, t) g(s) g(t) d s d t-\int_{0}^{a} \int_{0}^{u_{1}} \frac{\int_{0}^{u_{1}} f(r, t) g(r) d r}{\left\|g 1_{\left(0, u_{1}\right)}\right\|_{2}^{2}} g^{2}(s) g(t) d s d t \\
& -\int_{0}^{u_{2}} \int_{0}^{u_{1}} \frac{\int_{0}^{u_{2}} f(s, r) g(r) d r}{\left\|g 1_{\left(0, u_{2}\right)}\right\|_{2}^{2}} g(s) g^{2}(t) d s d t \\
& +\int_{0}^{u_{2}} \int_{0}^{u_{1}} \frac{\int_{0}^{u_{1}} \int_{0}^{u_{2}} f(r, v) g(r) g(v) d r d v}{\left\|g 1_{\left(0, u_{2}\right)}\right\|_{2}^{2}\left\|g 1_{\left(0, u_{1}\right)}\right\|_{2}^{2}} g^{2}(s) g^{2}(t) d s d t \\
= & 0 .
\end{aligned}
$$

Pour le point (ii) et (iii) ils se font par récurrence sur n après avoir remarqué que :

$$
\begin{array}{r}
T_{g}^{(\mathbf{u}, n)}(f)=T_{(g, 1)}^{\left(u_{1}\right)}\left[T_{(g, 2)}^{\left(u_{2}\right)} \cdots\left(T_{(g, n)}^{\left(u_{n}\right)} f\right)\right]=\cdots=T_{(g, n)}^{\left(u_{n}\right)}\left[T_{(g, n-1)}^{\left(u_{n-1}\right)} \cdots\left(T_{(g, 1)}^{\left(u_{1}\right)} f\right)\right] \\
S_{g}^{(\mathbf{u}, n)}(f)=S_{(g, 1)}^{\left(u_{1}\right)}\left[S_{(g, 2)}^{\left(u_{2}\right)} \cdots\left(S_{(g, n)}^{\left(u_{n}\right)} f\right)\right]=\cdots=S_{(g, n)}^{\left(u_{n}\right)}\left[S_{(g, n-1)}^{\left(u_{n-1}\right)} \cdots\left(S_{(g, 1)}^{\left(u_{1}\right)} f\right)\right]
\end{array}
$$

Comme conséquence immédiate de cette proposition nous avons le corollaire suivant :

Corollaire 2 Pour tout $n \geq 1$ et pour tout $\mathbf{u} \in C_{n}^{+}$on a :

1 -

$$
X_{n} \stackrel{\text { loi }}{=}\left\{X_{n}\left(S_{g}^{(\mathbf{u}, n)} f\right), f \in L^{(n)}\right\} \stackrel{\text { loi }}{=}\left\{X_{n, \mathbf{u}}^{g}\left(S_{g}^{(\mathbf{u}, n)} f\right), f \in L^{(n)}\right\}
$$

2- Les deux familles

$$
\left\{X_{n}\left(S_{g}^{(\mathbf{u}, n)} f\right), f \in L^{(n)}\right\} \text { et }\left\{X_{n}\left(g^{\otimes^{n}} 1_{R_{t}}\right), \mathbf{t} \in \partial R_{u}\right\}
$$

sont indépendantes.

3-

$$
X_{n, u}^{g} \stackrel{\text { loi }}{=}\left\{X_{n}\left(T_{g}^{(\mathbf{u}, n)} f\right), f \in L_{g}^{(\mathbf{u}, n)}\right\}
$$




\section{Preuve :}

1- Soit $f, h \in L^{(n)}$ on a :

$$
\begin{aligned}
\mathbb{E}\left(X_{n}\left(S_{g}^{(\mathbf{u}, n)} f\right) X_{n}\left(S_{g}^{(\mathbf{u}, n)} h\right)\right) & =\left(S_{g}^{(\mathbf{u}, n)} f, S_{g}^{(\mathbf{u}, n)} h\right)_{n}=(f, h)_{n} \\
\mathbb{E}\left(X_{n, u}^{g}\left(S_{g}^{(\mathbf{u}, n)} f\right) X_{n, u}^{g}\left(S_{g}^{(\mathbf{u}, n)} h\right)\right) & =\left(S_{g}^{(\mathbf{u}, n)} f, S_{g}^{(\mathbf{u}, n)} h\right)_{n}=(f, h)_{n}
\end{aligned}
$$

2- Soit $f \in L^{(n)}$ et $\mathbf{t} \in \partial R_{u}$ alors on a :

$$
\mathbb{E}\left(X_{n}\left(S_{g}^{(\mathbf{u}, n)} f\right) X_{n}\left(g^{\otimes^{n}} 1_{R_{t}}\right)\right)=\left(S_{g}^{(\mathbf{u}, n)} f, g^{\otimes^{n}} 1_{R_{t}}\right)_{n}=0
$$

où la dernière égalité est dûe au fait que $S_{g}^{(\mathbf{u}, n)} f$ appartient à $L_{g}^{(\mathbf{u}, n)}$

3 - il se fait de la même façon que le point 1 en utilisant la proposition 1 (ii) .

\section{Exemples :}

A- $n=u=1$; soit $B_{t}$ un mouvement brownien standard sur [0,1] Alors d'apès le corollaire 2 nous avons que :

$$
\begin{aligned}
B_{t}^{g, 1} & =B\left(S_{g}^{(1)} 1_{[0, t]}\right) \\
& =B_{t}-\int_{0}^{1} g(s) \int_{0}^{s \wedge t} \frac{g(r)}{\int_{r}^{1} g^{2}(r) d r} d B_{s} \\
& =B_{t}-\int_{0}^{1} g(s) \frac{\int_{s}^{1} g(r) d B_{r}}{\int_{s}^{1} g^{2}(r) d r} d s .
\end{aligned}
$$

où la dernière ligne est une conséquence du théorème de Fubini, est un mouvement brownien sur $[0,1]$ qui est indépendant de $\sigma\left(\int_{0}^{1} g(r) d B_{r}\right)$.

B- $n=2, \mathbf{u}=(1,1)$, Soit $B(s, t)$ un drap brownien sur $[0,1]^{2}$, Alors grâce toujours au corollaire 2, on a :

$$
\begin{aligned}
B^{(g, \mathbf{1})}(s, t)= & B\left(S_{g}^{\mathbf{1}, 2} 1_{[0, s] \times[0, t]}\right) \\
= & B(s, t)-\int_{0}^{t} \frac{g(r)}{\int_{r}^{1} g^{2}(r) d r} \int_{0}^{s} \int_{r}^{1} g(v) d B(u, v) d r-\int_{0}^{s} \frac{g(r)}{\int_{r}^{1} g^{2}(r) d r} \int_{0}^{t} \int_{r}^{1} g(u) d B(u, v) d r \\
& +\int_{0}^{s} \int_{0}^{t} \frac{g(a) g(b)}{\int_{a}^{1} g^{2}(z) d z \int_{b}^{1} g^{2}(z) d z} \int_{a}^{1} \int_{b}^{1} g(u) g(v) d B(u, v) d a d b .
\end{aligned}
$$

est un drap brownien sur $[0,1]^{2}$.

Notons qu'une décomposition non canonique de ce processus a été faite par Ouknine et Erraoui [3].

Dans ce qui suit nous allons définir le pont généralisé du drap brownien à n paramètre. Pour cela soit $n \geq 1$ et $\Gamma \in \mathcal{B}\left(C_{n}\right)$.

On désigne par $\mathcal{C}(\Gamma)$ la classe des fonctions déteministes continues à valeurs réelles qui s'annulent sur les axes.

Nous avons aussi besoin de la notation suivante pour la suite :

pour $\mathbf{t}=\left(t_{1}, \cdots, t_{n}\right)$ et $\mathbf{s}=\left(s_{1}, \cdots, s_{n}\right)$ de $C_{n}$, et $I \subset\{1, \cdots, n\}$, on désigne par $\mathbf{t}^{I} \mathbf{s}: l^{\prime}$ élément de $C_{n}$ constitué à la fois des $t_{i}$ et $s_{i}$ tels que; $\left(\mathbf{t}^{I} \mathbf{s}\right)_{i}=\left\{\begin{array}{cc}s_{i} & \text { si } i \in I \\ t_{i} & \text { si } i \notin I\end{array}\right.$

Définition :

Soit $\mathbf{u}=\left(u_{1}, \cdots, u_{n}\right) \in C_{n}^{+}$et $y \in \mathcal{C}\left(\partial R_{u}\right)$. 
Le pont généralisé du drap brownien indexé par $C_{n}$ et de point final y sur $\partial R_{u}$ est le processus :

$$
X_{n, \mathbf{u}}^{g, y}(\mathbf{t})=X_{n}\left(R_{g}^{(\mathbf{u}, n)} 1_{R_{\mathbf{t}}}\right)-\sum_{I \subset\{1, \cdots, n\}}(-1)^{|I|} y\left(\mathbf{t}^{I} \mathbf{u}\right) \prod_{i \in I} \frac{\int_{0}^{t_{i} \wedge u_{i}} g(r) d r}{\left\|g 1_{\left[0, u_{i}\right]}\right\|_{2}^{2}}, \forall \mathbf{t} \in C_{n}
$$

où $|I|$ est le cardinal de $I$.

La loi induite par le processus $X_{n, \mathbf{u}}^{g, y}$ sera notée $\mathbb{P}_{n, \mathbf{u}}^{g, y}$.

\section{Remarques :}

$\diamond$ On veut dire par le point final de $X_{n, \mathbf{u}}^{g, y}$ qu'il doit satisfaire l'égalité suivante :

$$
\int_{R_{\mathbf{t}}} g^{\otimes^{n}}(\mathbf{s}) d X_{n, \mathbf{u}}^{g, y}(\mathbf{s})=y(\mathbf{t}) \forall \mathbf{t} \in \partial R_{u} .
$$

$\diamond$ pour tout $A \in \mathcal{B}\left(C_{n}\right)$, l'application $y \longmapsto \mathbb{P}_{n, \mathbf{u}}^{g, y}(A)$ de $\mathcal{C}\left(\partial R_{u}\right)$ dans $[0,1]$ est mesurable.

\section{Exemples :}

A- $n=u=1$, soit $B_{t}$ un mouvement brownien standard sur [0,1] et y réel, Alors le processus :

$$
\begin{aligned}
B_{t}^{g, y} & =B\left(R_{g}^{(1,1)} 1_{[0, t]}\right)+\frac{\int_{0}^{t} g(r) d r}{\int_{0}^{1} g^{2}(r) d r} y \\
& =B_{t}-\frac{\int_{0}^{t} g(r) d r}{\int_{0}^{1} g^{2}(r) d r} \int_{0}^{1} g(r) d B_{r}+\frac{\int_{0}^{t} g(r) d r}{\int_{0}^{1} g^{2}(r) d r} y .
\end{aligned}
$$

est un pont généralisé sur [0,1] de point final $y$ (voir à ce sujet Alili [1] page 29). B- $n=2$ et $u=(a, b)$ soit $B(t, s)$ un drap brownien sur $[0,1]^{2}$ alors le processus

$$
\begin{aligned}
X_{(a, b)}^{g, 0}= & B\left(R_{g}^{(a, b)} 1_{[0, s] \times[0, t]}\right) \\
= & B(s, t)-\frac{\int_{0}^{s} g(r) d r}{\int_{0}^{a} g^{2}(r) d r} \int_{0}^{a} g(u) d B(u, t)-\frac{\int_{0}^{t} g(r) d r}{\int_{0}^{b} g^{2}(r) d r} \int_{0}^{a} g(v) d B(s, v) \\
& +\frac{\int_{0}^{s} g(r) d r \int_{0}^{t} g(r) d r}{\int_{0}^{a} g^{2}(r) d r \int_{0}^{b} g^{2}(r) d r} \int_{0}^{b} \int_{0}^{a} g(u) g(v) d B(u, v)
\end{aligned}
$$

est un pont généralisé du drap brownien de point final 0.

\section{Remarque}

la fonction $R_{g}^{(a, b)} 1_{[0, s] \times[0, t]}$ dans l'exemple précédent vérifie :

$$
d s \text { p.s } \int_{0}^{b} R_{g}^{(a, b)} 1_{[0, s] \times[0, t]}(s, v) g(v) d v=0, \text { et } d t \text { p.s } \int_{0}^{a} R_{g}^{(a, b)} 1_{[0, s] \times[0, t]}(u, t) g(u) d u=0
$$

et ceci grâce au fait que

$$
R_{g}^{(a, b)} 1_{[0, s] \times[0, t]}(s, v)=R_{g}^{(a)} 1_{[0, s]}(u) R_{g}^{(b)} 1_{[0, t]}(v) \text { et } \int_{0}^{u} R_{g}^{(u)} 1_{[0, s]}(r) g(r) d r=0
$$

nous allons maintenant donner deux résultats concernant le processus $X_{n, \mathbf{u}}^{g, 0}$ : 
Proposition 3 Soit $\mathbf{u} \in C_{n}^{+}$pour $n$ fixé on a :

1- les deux familles

$$
\left\{X_{n}\left(R_{g}^{(\mathbf{u}, n)} f\right), f \in L^{(n)}\right\} \text { et }\left\{X_{n}\left(g^{\otimes^{n}} 1_{R_{t}}\right), \mathbf{t} \in \partial R_{u}\right\}
$$

sont indépendantes et on a de plus

$$
\sigma\left\{X_{n}\left(f-R_{g}^{(\mathbf{u}, n)} f\right), f \in L^{(n)}\right\} \subset \sigma\left\{X_{n}\left(g^{\otimes^{n}} 1_{R_{t}}\right), \mathbf{t} \in \partial R_{u}\right\}
$$

2- l'application $y \longmapsto \mathbb{P} \mathbb{P}_{n, \mathbf{u}}^{g, y}, y \in \mathcal{C}\left(\partial R_{u}\right)$ est faiblement continue.

\section{Preuve}

1- Soit $f \in L^{(n)}$ et $\mathbf{t} \in \partial R_{u}$ alors on a

$$
\mathbb{E}\left(X_{n}\left(R_{g}^{(\mathbf{u}, n)} f\right) X_{n}\left(g^{\otimes^{n}} 1_{R_{t}}\right)\right)=\left(R_{g}^{(\mathbf{u}, n)} f, g^{\otimes^{n}} 1_{R_{t}}\right)_{n}=0
$$

où la dernière égalité est dûe au fait que $R_{g}^{(\mathbf{u}, n)} f$ appartient à $L_{g}^{(\mathbf{u}, n)}$.

Pour la deuxième partie de (1) il suffit de remarquer que $f-R_{g}^{(\mathbf{u}, n)} f$ s'écrit comme somme de fonctions de la forme

$$
\Psi\left(x_{1}, \cdots, x_{n}\right)=\varphi\left(x_{1}, \cdots, x_{n}\right) \prod_{i \in I \subset\{1, \cdots, n\}} g\left(x_{i}\right) 1_{\left[0, u_{i}\right]}\left(x_{i}\right)
$$

2- il s'agit de montrer que si $y_{N} \stackrel{\text { unif }}{\longrightarrow} y$ quand $N \longrightarrow \infty$ alors $\mathbb{P}_{n, \mathbf{u}}^{g, y_{N}} \stackrel{\text { faib }}{\longrightarrow} \mathbb{P}_{n, \mathbf{u}}^{g, y}$.

En effet, il suffit de remarquer que

$$
\sum_{I \subset\{1, \cdots, n\}}(-1)^{|I|} y_{N}\left(\mathbf{t}^{I} \mathbf{u}\right) \prod_{i \in I} \frac{\int_{0}^{t_{i} \wedge u_{i}} g(r) d r}{\left\|g 1_{\left[0, u_{i}\right]}\right\|_{2}^{2}} \longrightarrow \sum_{I \subset\{1, \cdots, n\}}(-1)^{|I|} y\left(\mathbf{t}^{I} \mathbf{u}\right) \prod_{i \in I} \frac{\int_{0}^{t_{i} \wedge u_{i}} g(r) d r}{\left\|g 1_{\left[0, u_{i}\right]}\right\|_{2}^{2}}
$$

uniformément quand $N \longrightarrow \infty$

L'exemple $\mathbf{B}$ et la remarque précédente nous poussent à se demander quel est le lien entre l'espace gaussien du processus $X_{n, \mathbf{u}}^{g, 0}$ qu'on va noter $\mathcal{H}_{n}^{g}$ et le sous espace de l'espace gaussien de $X_{n}$ défini par :

$$
\begin{aligned}
\mathcal{G}_{n}:=\{G \text { v.a, } \quad & G \in \mathcal{H}\left(X_{n}\right) \text { telle que } \\
& \left.G \perp \sigma\left(\int_{R_{t}^{I}} g^{\otimes}(\mathbf{v}) d X_{n}(\mathbf{s}, \mathbf{v}), \forall I \subset\{1, \cdots, n\} \forall \mathbf{t} \in \partial R_{u} \text { et } \forall \mathbf{s} \in R_{t}^{I^{c}}\right)\right\}
\end{aligned}
$$

avec $R_{t}^{J}=\prod_{i \in J}\left[0, t_{i}\right]$

la réponse est donnée dans la proposition suivante :

Proposition 4 Soit $\mathbf{u}=\left(u_{1}, \cdots, u_{n}\right) \in C_{n}$ fixé,

1- une variable $G$ appartient $\grave{a} \mathcal{G}_{n}$ si et seulement si :

$$
G=\int_{R_{u}} f(\mathbf{r}) X_{n}(d \mathbf{r})
$$

où $f \in L^{2}\left(R_{u}, d \lambda_{n}\right)$ et vérifie en outre

$$
\forall I \subset\{1, \cdots, n\} \forall \mathbf{t} \in \partial R_{u} d \lambda_{\left|I^{c}\right|}(\mathbf{s}) p . s, \int_{R_{t}^{I}} f(\mathbf{s}, \mathbf{v}) g^{\otimes^{|I|}}(\mathbf{v}) d \lambda_{|I|}(\mathbf{v})=0
$$

2-

$$
\mathcal{G}_{n}=\mathcal{H}_{n}^{g}
$$




\section{Preuve :}

$1-G \in \mathcal{G}_{n}$ si et seulement si elle est de la forme

$$
\int_{\mathbb{R}_{+}^{n}} f(\mathbf{r}) X_{n}(d \mathbf{r})
$$

avec $f \in L^{2}\left(\mathbb{R}_{+}^{n}\right)$ et de plus :

$$
\forall I \subset\{1, \cdots, n\} \forall \mathbf{t} \in \partial R_{u} \int_{R_{t}^{I^{c}} \times R_{t}^{I}} f(\mathbf{s}, \mathbf{v}) g^{\otimes^{|I|}}(\mathbf{v}) d \lambda_{n}(\mathbf{s}, \mathbf{v})=0
$$

ce qui entraine (9).

2- il est clair que $\mathcal{H}_{n}^{g} \subset \mathcal{G}_{n}$ puisque $X_{n, \mathbf{u}}^{g, 0}(\mathbf{t})=X_{n}\left(R_{g}^{(\mathbf{u}, n)} 1_{R_{\mathbf{t}}}\right)$ et la fonction $R_{g}^{(\mathbf{u}, n)} 1_{R_{\mathbf{t}}}$ vérifie la condition (9).

pour l'autre inclusion, si $G \in \mathcal{G}_{n}$ alors

$$
G=\int_{R_{u}} f(\mathbf{r}) X_{n}(d \mathbf{r})=\int_{R_{u}} R_{g}^{(\mathbf{u}, n)} f(\mathbf{r}) X_{n}(d \mathbf{r})=\int_{R_{u}} f(\mathbf{r}) X_{n, \mathbf{u}}^{g, 0}(d \mathbf{r})
$$

ceci car $f$ vérifie (9), donc appartient à $L_{g}^{(\mathbf{u}, n)}$ et on conclure par la proposition 3 (i).

\section{Représentation Chaotique et pont généralisé du drap brow- nien}

\subsection{Polynômes d'Hermite et intégrales multiples}

Considèrons, pour $n \geq 1$ et $\mathbf{u} \in C_{n}^{+}$, un processus gaussien isonormal $X_{n}$ dans $L^{(n)}$ et une famille $X_{n, \mathbf{u}}^{g, 0}$ telle que

$$
\left\{X_{n, \mathbf{u}}^{g, 0}(f), f \in L^{(n)}\right\}:=\left\{X_{n}\left(T_{g}^{(\mathbf{u}, n)} f\right), f \in L^{(n)}\right\}
$$

c-à-d $\left\{X_{n, \mathbf{u}}^{g, 0}(f), f \in L^{(n)}\right\}$ est l'espace gaussien engendré par le processus $X_{n}\left(T_{g}^{(\mathbf{u}, n)} 1_{R_{t}}\right)$, $\mathbf{t} \in C_{n}$, ayant la loi du pont généralisé de drap brownien de point final 0 sur $\partial R_{u}$.

Notre but dans ce paragraphe est d'établir une représentation Chaotique pour le processus $X_{n, \mathbf{u}}^{g, 0}$. Pour cela on considère les polynômes d'Hermite $H_{k}$ définis par

$$
H_{k}(x)=\frac{(-1)^{k}}{k !} \exp \left(\frac{x^{2}}{2}\right) \frac{d^{k}}{d x^{k}} \exp \left(-\frac{x^{2}}{2}\right) \quad k \geq 1 .
$$

nous començons d'abord par le résultat suivant :

Proposition 5 Soit $\mathcal{H}^{g}$ l'ensemble des fonctions de carrée intégrables du processus $X_{n, \mathbf{u}}^{g, 0}$ et $\mathcal{H}_{k}^{g} k \geq 0$ la fermeture dans $L^{2}$ de l'espace vectoriel engendré par la classe :

$$
\left\{H_{k}\left(X_{n, \mathbf{u}}^{g, 0}(f)\right), \quad f \in L_{g}^{(\mathbf{u}, n)},\|f\|_{n}=1\right\}
$$

avec $\mathcal{H}_{0}^{g}=\mathcal{R} ;$ Alors

$$
\mathcal{H}^{g}=\bigoplus_{k} \mathcal{H}_{k}^{g}
$$


la preuve est immédiate (voir [7] théorème 1.1.1).

Maintenant, soit $\mathcal{H}$ l'ensemble des fonctions de carrée intégrables du processus $X_{n, \mathbf{u}}$ et $\mathcal{H}_{k} k \geq 0$ la fermeture dans $L^{2}$ de l'espace vectoriel engendré par la classe :

$$
\left\{H_{k}\left(X_{n, \mathbf{u}}(f)\right), \quad f \in L^{(n)},\|f\|_{n}=1\right\}
$$

avec $\mathcal{H}_{0}=\mathcal{R}$, et puisque $X_{n, \mathbf{u}}^{g, 0}(f)=X_{n, \mathbf{u}}\left(T_{g}^{(\mathbf{u}, n)} f\right)$ où $T_{g}^{(\mathbf{u}, n)}$ est un isomorphisme de $L_{g}^{(\mathbf{u}, n)}$ dans $L^{(n)}$, alors on a :

$$
\begin{aligned}
\mathcal{H}_{k}^{g} & =\mathcal{H}_{k} \\
\mathcal{H}^{g} & =\mathcal{H}
\end{aligned}
$$

Or, on sait d'après Nualart [7], et puisque $X_{n, \mathbf{u}}$ est un processus isonoraml dans $L^{(n)}$, que $\mathcal{H}_{k}$ coincide avec la classe des intégrales multiples de la forme

$$
I_{k}^{X}(f)=\int_{C_{n k}} f\left(\mathbf{t}^{(1)}, \cdots, \mathbf{t}^{(k)}\right) X_{n, \mathbf{u}}\left(d \mathbf{t}^{(1)}\right) \cdots X_{n, \mathbf{u}}\left(d \mathbf{t}^{(k)}\right)
$$

avec $\mathbf{t}^{(i)}:=\left(t_{(i-1) n+1} \cdots, t_{i n}\right) i=1 \cdots, k$, et la fonction $f$ appartient à $L^{(n k)}$ et elle est symétrique dans le sens suivant : pour tout $\left(t_{1}, \cdots, t_{n k}\right) \in C_{n k}$ et pour tout $\mathbf{t}^{(i)} i=1 \cdots, k$ on a :

$$
f\left(\mathbf{t}^{(1)}, \cdots, \mathbf{t}^{(k)}\right)=f\left(\mathbf{t}^{(\pi(1))}, \cdots, \mathbf{t}^{(\pi(k))}\right)
$$

pour toute permutation $\pi$ de l'ensemble $\{1, \cdots, k\}$. on veut montrer un résultat similaire pour le processus $X_{n, \mathbf{u}}^{g, 0}$.

Considérons la notation suivante : pour tout $n, k \geq 1$ et tout $\mathbf{t}=\left(t_{1}, \cdots, t_{n}\right) \in C_{n}$ on note par $t^{k}$ le vecteur symétrique

$$
(\underbrace{t_{1}, \cdots, t_{n} ; t_{1}, \cdots, t_{n} ; \cdots ; t_{1}, \cdots, t_{n}}_{k \text { fois }}) \in C_{n k}
$$

Alors on a le lemme suivant :

Lemme 6 pour tout entier $k>0$, la famille des fonctions définies sur $C_{n k}$ par :

$$
f\left(t_{1} \cdots, t_{n k}\right)=f_{1}\left(\mathbf{t}^{(1)}\right) \cdots f_{k}\left(\mathbf{t}^{(k)}\right)
$$

avec $\mathbf{t}^{(i)}:=\left(t_{(i-1) n+1} \cdots, t_{i n}\right) i=1 \cdots, k$, et les $f_{i}$ sont toutes dans $L_{g}^{(u, n)}$ et vérifient

$$
\left(f_{i}, f_{j}\right)_{n}=0, \quad \forall i \neq j
$$

définissent un ensemble total de $L_{g}^{\left(\mathbf{u}^{k}, n k\right)}$.

\section{Preuve :}

Preuve :
Il est evident que les fonctions de la forme (11) appartiennent à $L_{g}^{\left(\mathbf{u}^{k}, n k\right)}$. Soit $h \in L_{g}^{\left(\mathbf{u}^{k}, n k\right)}$ telle que

$$
(h, f)_{n k}=0
$$

pour tout $f$ de la forme (11), on doit montrer que $h=0$.

on a :

$$
(f, h)_{n k}=\left(T_{g}^{\left(\mathbf{u}^{k}, n k\right)} f, T_{g}^{\left(\mathbf{u}^{k}, n k\right)} h\right)_{n k}=0
$$


or $T_{g}^{\left(\mathbf{u}^{k}, n k\right)} f=T_{g}^{(\mathbf{u}, n)} f_{1} \cdots T_{g}^{(u, n)} f_{k}$ et

$$
\left(T_{g}^{(\mathbf{u}, n)} f_{i}, T_{g}^{(\mathbf{u}, n)} f_{j}\right)_{n}=0, \quad \forall i \neq j
$$

De plus, tout élément de $L^{(n k)}$ de la forme

$$
\begin{aligned}
l\left(t_{1}, \cdots, t_{n k}\right) & =l_{1}\left(\mathbf{t}^{(1)}\right) \cdots l_{k}\left(\mathbf{t}^{(k)}\right) \\
\text { où } l_{i} & \in L^{(n)} ; \text { telles que }\left(l_{i}, l_{j}\right)_{n}=0, \forall i \neq j
\end{aligned}
$$

peut être écrit sous la forme $l=T_{g}^{\left(\mathbf{u}^{k}, n k\right)} f=T_{g}^{(\mathbf{u}, n)} f_{1} \cdots T_{g}^{(\mathbf{u}, n)} f_{k}$ où $f$ et les $f_{i}$ sont comme dans (11), ceci est dû au fait que la classe des fonctions de la forme (12) est total dans $L^{(n k)}$ (voir [7]). Ce qui entraine que

et par suite $h=0$.

$$
T_{g}^{\left(\mathbf{u}^{k}, n k\right)} h=0
$$

Pour la suite pour tout $k$, on va noter la classe des fonctions de la forme (11) par $\varepsilon_{n}^{k}$, et pose pour tout $f \in \varepsilon_{n}^{k}:$

$$
\begin{aligned}
I_{k}^{X^{g, 0}}(f) & :=\prod_{i=1}^{k} X_{n, \mathbf{u}}^{g, 0}\left(f_{i}\right) \\
& \stackrel{\text { not }}{=} \int_{C_{n k}} f\left(t^{(1)}, \cdots, t^{(k)}\right) X_{n, \mathbf{u}}^{g, 0}\left(d t^{(1)}\right) \cdots X_{n, \mathbf{u}}^{g, 0}\left(d t^{(k)}\right) .
\end{aligned}
$$

Le symétrisé de $f$ dans $C_{n k}$ est donné par

$$
\widetilde{f}\left(\mathbf{t}^{(1)}, \cdots, \mathbf{t}^{(k)}\right)=\frac{1}{k !} \sum_{\pi \in \Im_{k}} f\left(\mathbf{t}^{(\pi(1))}, \cdots, \mathbf{t}^{(\pi(k))}\right)
$$

où $\pi$ parcourt l'ensemble des permutations sur $\{1, \cdots, k\}$ noté $\Im_{k}$.

Si $f \in \varepsilon_{n}^{k}$ et $g \in \varepsilon_{n}^{k^{\prime}}$ alors on a :

$$
\begin{aligned}
\mathbb{E}\left(I_{k}^{X^{g, 0}}(f) I_{k^{\prime}}^{X^{g, 0}}(g)\right) & =\mathbb{E}\left(I_{k}^{X}\left(T_{g}^{\left(\mathbf{u}^{k}, n k\right)} f\right) I^{X_{k^{\prime}}}\left(T_{g}^{\left(\mathbf{u}^{k^{\prime}}, n k^{\prime}\right)} g\right)\right) \\
& =k !\left(\widetilde{T_{g}^{\left(\mathbf{u}^{k}, n k\right)}} f, T_{g}^{\left(\mathbf{u}^{k^{\prime}}, n k^{\prime}\right)} g\right)_{n k} \delta_{k, k^{\prime}} \\
& =k !\left(T_{g}^{\left(\mathbf{u}^{k}, n k\right)} \widetilde{f}, T_{g}^{\left(\mathbf{u}^{k^{\prime}}, n k^{\prime}\right)} \widetilde{g}\right)_{n k} \delta_{k, k^{\prime}} \\
& =k !(\widetilde{f}, \widetilde{g})_{n k} \delta_{k, k^{\prime}}
\end{aligned}
$$

avec $\delta$ est le symbol de Kronecker, notons que la dernière égalité est dûe au fait que $\widetilde{f}, \widetilde{g} \in L_{g}^{\left(\mathbf{u}^{k}, n k\right)}$ puisque $f, g \in \varepsilon_{n}^{k}$.

par conséquent on peut étendre le domaine de $I_{k}^{X^{g, 0}}$ à $L^{\left(\mathbf{u}^{k}, n k\right)}$ en définissant $I_{k}^{X^{g, 0}}(f)$ pour les éléments de $f \in L_{g}^{\left(\mathbf{u}^{k}, n k\right)}$ comme limite dans $L^{2}$ de la suite $I_{k}^{X^{g, 0}}\left(f_{N}\right)$, où $f_{N}$ appartient à l'espace vectoriel engendré par $\varepsilon_{n}^{k}$ pour tout $N$, et $f_{N}$ converge, quand $N$ tend vers l'infini, vers $f$ dans $L_{g}^{\left(\mathbf{u}^{k}, n k\right)}$. On a en outre les égalités suivantes :

$$
\begin{aligned}
I_{k}^{X^{g, 0}}(f) & =I_{k}^{X}\left(T_{g}^{\left(\mathbf{u}^{k}, n k\right)} f\right), \quad \forall f \in L_{g}^{\left(\mathbf{u}^{k}, n k\right)} \\
I_{k}^{X}(f) & =I_{k}^{X^{g, 0}}\left(S_{g}^{\left(\mathbf{u}^{k}, n k\right)} f\right), \quad \forall f \in L^{(n k)} \\
I_{k}^{X^{g, 0}}(f) & =I_{k}^{X^{g, 0}}(\widetilde{f}), \quad \forall f \in L_{g}^{\left(u^{k}, n k\right)} \\
\mathbb{E}\left(I_{k}^{X^{g, 0}}(f) I_{k^{\prime}}^{X^{g, 0}}(g)\right) & =k !(\widetilde{f}, \widetilde{g}) \delta_{k, k^{\prime}}, \quad \forall f \in L_{g}^{\left(\mathbf{u}^{k}, n k\right)}, g \in L_{g}^{\left(\mathbf{u}^{k^{\prime}}, n k^{\prime}\right)}
\end{aligned}
$$


Ceci va nous permettre d'énoncer le résultat suivant :

Proposition 7 Soit $F$ un élément de $\mathcal{H}=\mathcal{H}^{g}$ les conditions suivantes sont équivalentes :

1- F appartient à $\mathcal{H}=\mathcal{H}^{g}$.

2- il existe une fonction $f \in L^{(n k)}$, symétrique au sens de (10) telle que :

$$
F=I_{k}^{X}(f)
$$

3-il existe une fonction $g \in L_{g}^{\left(\mathbf{u}^{k}, n k\right)}$, symétrique au sens de (10) telle que :

$$
F=I_{k}^{X^{g, 0}}(g)
$$

de plus $f$ et $g$ sont liées par la relation suivante:

$$
\begin{aligned}
& f=T_{g}^{\left(\mathbf{u}^{k}, n k\right)} g \\
& g=S_{g}^{\left(\mathbf{u}^{k}, n k\right)} f
\end{aligned}
$$

Comme conséquence immédiate de ce corollaire on a la représentation Chotique du processus $X_{n, \mathbf{u}}^{g, 0}$.

Corollaire 8 Pour tout $F \in \mathcal{H}\left(=\mathcal{H}^{g}\right)$, il existe deux suites de fonctions $\left(f_{N}, N \geq 1\right)$ et $\left(g_{N}, N \geq 1\right)$ dans $L^{(n N)}$ et $L_{g}^{\left(\mathbf{u}^{N}, n N\right)}$ respectivement, symétriques au sens de (10) telles que :

$$
\begin{aligned}
F & =\mathbb{E}(F)+\sum_{N \geq 1} I_{N}^{X^{g, 0}}\left(g_{N}\right) \\
& =\mathbb{E}(F)+\sum_{N \geq 1} I_{N}^{X}\left(f_{N}\right)
\end{aligned}
$$

De plus $f_{N}$ et $g_{N}$ sont liées par la relation suivante:

$$
\begin{aligned}
& f_{N}=T_{g}^{\left(\mathbf{u}^{k}, n k\right)} g_{N} \\
& g_{N}=S_{g}^{\left(\mathbf{u}^{k}, n k\right)} f_{N}
\end{aligned}
$$

Pour tout $N \geq 1$.

\subsection{Formule de Stroock}

Dans ce paragraphe nous allons donner une formule explicite pour les $g_{N}$ qui figurent dans le corollaire 8 .

Pour cela, on considère $\mathcal{S}\left(X_{n}\right)$ la classe des fonctions régulières de $X_{n}$ ie : les fonctionnelles de $X_{n}$ de la forme

$$
F\left(X_{n}\right)=f\left(X_{n}\left(h_{1}\right), \cdots, X_{n}\left(h_{m}\right)\right)
$$

avec $h_{i} \in L^{(n)} \quad i=1, \cdots, m$ et $f \in \mathcal{C}_{b}^{\infty}\left(\mathcal{R}^{m}\right)$ : l'ensemble des fonctions bornées, indéfiniment différentiables et à dérivées de tout ordre bornées.

De même, et puisque $X_{n, \mathbf{u}}^{g, 0}(f)=X_{n, \mathbf{u}}^{g, 0}\left(R_{g}^{(\mathbf{u}, n)} f\right)=X_{n}\left(T_{g}^{(\mathbf{u}, n)} f\right)$, on peut définir la classe des fonctions régulières de $X_{n, \mathbf{u}}^{g, 0}$, ie : les fonctionnelles de $X_{n, \mathbf{u}}^{g, 0}$ de la forme

$$
G\left(X_{n, \mathbf{u}}^{g, 0}\right)=g\left(X_{n, \mathbf{u}}^{g, 0}\left(z_{1}\right), \cdots, X_{n, \mathbf{u}}^{g, 0}\left(z_{m}\right)\right)
$$

avec $z_{i} \in L_{g}^{(\mathbf{u}, n)}$ et $g \in \mathcal{C}_{b}^{\infty}\left(\mathcal{R}^{m}\right)$.

Rappelons que pour $F \in \mathcal{S}\left(X_{n}\right)$, la dérivée de Malliavin de $F$ est donnée par :

$$
D_{\mathbf{t}} F\left(X_{n}\right)=\sum_{i=1}^{m} \frac{\partial}{\partial x_{i}} f\left(X_{n}\left(h_{1}\right), \cdots, X_{n}\left(h_{m}\right)\right) h_{i}(\mathbf{t}), \quad \mathbf{t} \in C_{n}
$$


De même, pour $F \in \mathcal{S}\left(X^{g, 0}\right)$ on a :

$$
D_{\mathbf{t}} F\left(X_{n, \mathbf{u}}^{g, 0}\right)=\sum_{i=1}^{m} \frac{\partial}{\partial x_{i}} f\left(X_{n, \mathbf{u}}^{g, 0}\left(z_{1}\right), \cdots, X_{n, \mathbf{u}}^{g, 0}\left(z_{m}\right)\right) z_{i}(\mathbf{t}), \quad \mathbf{t} \in C_{n}
$$

il est clair que

$$
\begin{aligned}
D F\left(X_{n}\right) & =T_{g}^{(\mathbf{u}, n)} \operatorname{DF}\left(X_{n, \mathbf{u}}^{g, 0}\right) \\
D F\left(X_{n, \mathbf{u}}^{g, 0}\right) & =S_{g}^{(\mathbf{u}, n)} \operatorname{DF}\left(X_{n}\right)
\end{aligned}
$$

Maintenant, soit $k \geq 1$ et $\mathbf{t}=\left(t_{1}, \cdots, t_{n k}\right) \in C_{n k}$ et $\mathbf{t}^{(i)}=\left(t_{(i-1) n+1}, \cdots, t_{i n}\right) i=1 \cdots, k$, on peut alors définir la kiéme dérivée de Malliavin de tout $F \in \mathcal{S}\left(X_{n, \mathbf{u}}^{g, 0}\right)$ par

$$
D_{\mathbf{t}}^{k} F\left(X_{n, \mathbf{u}}^{g, 0}\right)=D_{t_{1}, \cdots, t_{n k}}^{k} F\left(X_{n, \mathbf{u}}^{g, 0}\right)=D_{\mathbf{t}^{(1)}} D_{\mathbf{t}^{(2)}} \cdots D_{\mathbf{t}^{(k)}} F\left(X_{n, \mathbf{u}}^{g, 0}\right)
$$

Notons que $D^{k} F\left(X_{n}\right)$ et $D^{k} F\left(X_{n, \mathbf{u}}^{g, 0}\right)$ sont symétriques au sens de (10) et sont liées par la relation suivante :

$$
\begin{aligned}
D^{k} F\left(X_{n}\right) & =T_{g}^{\left(\mathbf{u}^{k}, n k\right)} D^{k} F\left(X_{n, \mathbf{u}}^{g, 0}\right) \\
D^{k} F\left(X_{n, \mathbf{u}}^{g, 0}\right) & =S_{g}^{\left(\mathbf{u}^{k}, n k\right)} D^{k} F\left(X_{n}\right)
\end{aligned}
$$

Nous allons maintenant introduire la norme suivante dans $\mathcal{S}$

$$
\begin{aligned}
\|F\|_{(k, n)} & :=\left[\mathbb{E}\left(F^{2}\right)+\sum_{i=1}^{k} \mathbb{E}\left(\left\|D^{i} F\left(X_{n}\right)\right\|_{n i}^{2}\right)\right]^{\frac{1}{2}} \\
& =\left[\mathbb{E}\left(F^{2}\right)+\sum_{i=1}^{k} \mathbb{E}\left(\left\|D^{i} F\left(X_{n, \mathbf{u}}^{g, 0}\right)\right\|_{n i}^{2}\right)\right]^{\frac{1}{2}}
\end{aligned}
$$

La fermeture de $\mathcal{S}$ par rapport à cette norme sera notée par $\mathbb{D}^{k}$ et on a pour tout $F \in \mathbb{D}^{k}$

$$
\begin{aligned}
& D^{k} F\left(X_{n}\right)=\quad T_{g}^{\left(\mathbf{u}^{k}, n k\right)} D^{k} F\left(X_{n, \mathbf{u}}^{g, 0}\right) \\
& D^{k} F\left(X_{n, \mathbf{u}}^{g, 0}\right)=S_{g}^{\left(\mathbf{u}^{k}, n k\right)} D^{k} F\left(X_{n}\right)
\end{aligned}
$$

Cela nous donne le résultat principal de ce paragraphe (Formule de Stroock)

Proposition 9 Soit $F \in \bigcap_{N \geq 1} \mathbb{D}^{N}$ et soient $\left(f_{N}, N \geq 1\right)$ et $\left(g_{N}, N \geq 1\right)$ où $f_{N} \in L^{(n N)}$ et $g_{N} \in L_{g}^{\left(\mathbf{u}^{N}, n N\right)}$ pour tout $N \geq 1$, deux suites symétriques au sens de (10). Alors on a

$$
\begin{aligned}
f_{N}\left(t_{1}, \cdots, t_{n N}\right) & =\frac{1}{N !} \mathbb{E}\left(D_{t_{1}, \cdots, t_{n N}}^{N} F\left(X_{n}\right)\right) \\
g_{N}\left(t_{1}, \cdots, t_{n N}\right) & =\frac{1}{N !} \mathbb{E}\left(D_{t_{1}, \cdots, t_{n N}}^{N} F\left(X_{n, \mathbf{u}}^{g, 0}\right)\right)
\end{aligned}
$$

La preuve est une simple conséquence des calculs précédents 


\section{Références}

[1] Alili L. (2000). Canonical decompositions of certain generalized Brownian bridges. In : Elect. Comm. in Probab. 7 (2002) 27 - 35.

[2] Elhssaini Y., Ouknine Y. (2004). Formule explicite des Chaos d'espace-temps généralisés.Submitted.

[3] Erraoui M., Ouknine Y. (2003) Une représentation non-canonique du drap brownien. Publication Mathématique (To appear)

[4] Gosselin P.,Wurzbacher T. (1997). An Itô type isometry for loops in $\mathbf{R}^{d}$ via the Brownian bridge. In Azema J., Emery M. and Yor M. (eds), Séminaire de Probabilités XIII, pp. 225-231. SpringerVerlag, Berlin.

[5] Hibino Y., Hitsuda M., Muraoka H. (1997). Construction of noncanonical representations of a Brownian motion, Hiroshima Math. J. 27 (1997), 439-448

[6] Jeulin T., Chaleyat-Maurel M.(1982). Grossissement Gaussien de la Filtration Brownienne, in : Lecture Notes in Mathematics 1118, Springer, Berlin, 59-107

[7] Nualart D.(1995). The Malliavin Calculus and Related Topics. Springer-Verlag, Berlin. 